\title{
A 13-year survey of bacteraemia due to $\beta$-haemolytic streptococci in a Danish county
}

\author{
B. KRISTENSEN* and H. C. SCHØNHEYDER
}

Department of Clinical Microbiology, Aalborg Hospital, DK-9000 Aalborg, Denmark

\begin{abstract}
Summary. During 1981-1993, 229 episodes of bacteraemia due to $\beta$-haemolytic streptococci of groups A, B, C and G were diagnosed in the County of Northern Jutland, Denmark. The annual rates for bacteraemia were quite constant during the 13-year period for each streptococcal group. Group A streptococcal (GAS) bacteraemia was the most frequent, comprising $1.4 \%$ of all bacteraemias. The incidence of GAS bacteraemia was $1.8 / 100000$ / year in children $<5$ years of age and $4 \cdot 7 / 100000 /$ year in patients $>60$ years old. With the notable exception of group B streptococcal (GBS) bacteraemia in neonates, $\beta$-haemolytic streptococci of groups B, C (GCS) and G (GGS) were isolated mostly from elderly patients. Except for GBS bacteraemia in neonates, approximately one-third of the bacteraemias in each group was nosocomially acquired. Predisposing factors included operative procedures in GAS and GCS bacteraemia, and diabetes mellitus in GBS bacteraemia. The skin was the most common primary focus in GAC, GCC and GGS bacteraemias, whereas the urinary tract was the commonest focus in GBS bacteraemia in adults. The mortality rates in GAS, GCS, GGS, and adult GBS bacteraemia were $23 \%, 16 \%, 17 \%$ and $19 \%$, respectively. Of the 23 fatal cases of GAS bacteraemia, $57 \%$ died within $24 \mathrm{~h}$ after blood cultures had been obtained.
\end{abstract}

\section{Introduction}

$\beta$-Haemolytic streptococci continue to be significant pathogens and in the late 1980s an increase in group A streptococcal (GAS) infections in the Scandinavian countries and in the USA was reported. ${ }^{1-4}$ Attention has been drawn to a cluster of cases of necrotising fasciitis due to GAS in Great Britain, ${ }^{5}$ and there has been an increase of GAS bacteraemia again in Norway. ${ }^{6}$ Furthermore, reports have indicated increases of both group B streptococcal (GBS) invasive disease in adults ${ }^{7}$ and group G streptococcal (GGS) bacteraemias. $^{8}$

In this study, the blood isolates in a Danish county obtained during a 13-year period (1981-1993) were reviewed retrospectively to assess any major trends in the occurrence of $\beta$-haemolytic streptococci of groups $\mathrm{A}, \mathrm{B}, \mathrm{C}$ and $\mathrm{G}$ in a defined study population. The aim of the study was to elucidate the epidemiology of bacteraemia with $\beta$-haemolytic streptococci of groups $\mathrm{A}, \mathrm{B}, \mathrm{C}$ and $\mathrm{G}$, in association with the clinical picture and outcome.

Received 9 Jan. 1995; accepted 12 Jan. 1995.

* Present address and address for correspondence: The National Centre of Hygiene, Statens Seruminstitut, Artillerivej 5, DK-2300 Copenhagen, Denmark.

\section{Materials and methods}

The County of Northern Jutland had a mean population of 483000 during the observation period. There were nine local hospitals and two referral hospitals with specialised medical and surgical departments, including neurosurgery, gynaecology and paediatrics. The Department of Clinical Microbiology of Aalborg Hospital performed all the blood cultures during the study period. From 1 Jan. 1981 to 31 March 1992, a multiple-tube blood culture system was used ${ }^{9}$ and from 1 April 1992 to 31 Dec. 1993, a broth culture system. ${ }^{10}$ All streptococci that were $\beta$ haemolytic on horse blood $5 \%$ agar were sent to the Streptococcal Laboratory, Statens Seruminstitut, Copenhagen, for confirmation of grouping, T-typing of GAS and serotyping of GBS. The records of all patients with isolates belonging to groups $A, B, C$ or $G$ were reviewed retrospectively in regard to whether the bacteraemia was nosocomially or community acquired, whether there was underlying disease, the site of the primary focus and the outcome. Centers for Disease Control (CDC) define nosocomial bacteraemias as those cases in which no evidence of infection is present on admission to hospital. ${ }^{11}$ Cases related either to previous admission to hospital or treatment, and infections in newborns associated with 
vaginal delivery were included. ${ }^{11}$ The primary focus was assessed on the basis of clinical findings on the day of blood culture, and was considered to be verified bacteriologically if $\beta$-haemolytic streptococci of the same group were found at a relevant anatomical site. Death was considered to be related to the streptococcal bacteraemia if it occurred within 7 days after positive blood cultures were obtained. The annual incidences were calculated on the basis of the age-related population statistics for the County of Northern Jutland.

Mann-Whitney and $\chi^{2}$ tests were used to assess differences between observed rates.

\section{Results}

During the 13-year survey there were 6849 bacteraemias identified in the County of Northern Jutland: 249 episodes of $\beta$-haemolytic streptococcal infection were recorded in 216 patients. Thirteen patients were excluded from further investigation either because the isolate was lost on subculture (one patient), the hospital record was not available (one patient), the bacteriological diagnosis was Streptococcus anginosus (six patients) and group F streptococci (two patients), or a streptococcal group identification was not obtained (three patients). In seven patients with recurrent bacteraemia with $\beta$ haemolytic streptococci, only the first episode was included. This resulted in 229 bacteraemic episodes available for study. Streptococci were associated with polymicrobial bacteraemia in 21 patients; four patients had GAS, 10 GBS, three group C streptococci (GCS), and four GGS bacteraemia.

The annual number of $\beta$-haemolytic streptococcal bacteraemia, and the total number of bacteraemias, are shown in table I. GAS accounted for $43 \%$ of all $\beta$-haemolytic streptococcal bacteraemias and GBS, GGS, and GCS for $31 \%, 16 \%$ and $10 \%$, respectively.

Table I. Number and distribution of $\beta$-haemolytic streptococcal bacteraemias in the County of Northern Jutland 1981-1993

\begin{tabular}{|c|c|c|c|c|c|}
\hline \multirow{2}{*}{ Year } & \multicolumn{4}{|c|}{$\begin{array}{c}\text { Number of streptococcal } \\
\text { bacteraemias }\end{array}$} & \multirow{2}{*}{$\begin{array}{c}\text { Total } \\
\text { number of } \\
\text { bacteraemic } \\
\text { episodes }\end{array}$} \\
\hline & GAS & GBS & GCS & GGS & \\
\hline 1981 & 2 & 3 & 1 & 1 & 443 \\
\hline 1982 & 3 & 4 & 4 & 1 & 454 \\
\hline 1983 & 7 & 7 & 1 & 1 & 474 \\
\hline 1984 & 7 & 1 & 2 & 4 & 503 \\
\hline 1985 & 7 & 12 & 1 & 2 & 516 \\
\hline 1986 & 11 & 6 & 3 & 1 & 474 \\
\hline 1987 & 6 & 5 & 1 & 1 & 516 \\
\hline 1988 & 10 & 3 & 2 & 4 & 521 \\
\hline 1989 & 12 & 5 & 0 & 5 & 495 \\
\hline 1990 & 7 & 6 & 0 & 4 & 514 \\
\hline 1991 & 5 & 5 & 3 & 4 & 505 \\
\hline 1992 & 11 & 6 & 2 & 4 & 676 \\
\hline 1993 & 11 & 7 & 4 & 4 & 758 \\
\hline Total & 99 & 70 & 24 & 36 & 6849 \\
\hline
\end{tabular}

Table II. Epidemiological characteristics of patients with $\beta$ haemolytic streptococcal bacteraemia in the County of Northern Jutland 1981-1993

\begin{tabular}{|c|c|c|c|c|c|}
\hline \multirow{2}{*}{ Parameter } & \multirow{2}{*}{ GAS } & \multicolumn{2}{|c|}{ GBS } & \multirow{2}{*}{ GCS } & \multirow{2}{*}{ GGS } \\
\hline & & Neonatal & Adults & & \\
\hline \multicolumn{6}{|l|}{ Sex } \\
\hline Male & 49 & 21 & 20 & 11 & 25 \\
\hline Female & 50 & 11 & 18 & 13 & 11 \\
\hline \multicolumn{6}{|l|}{ Age (years) } \\
\hline Median & $61 \cdot 8$ & $1 \cdot 3^{*}$ & 67 & 57.5 & 65 \\
\hline Range & $0-91$ & $0-56^{*}$ & $16-91$ & $18-89$ & $22-85$ \\
\hline $\begin{array}{l}\text { Nosocomial } \\
\text { cases }\end{array}$ & 38 & 28 & 15 & 7 & 12 \\
\hline Died & 23 & 9 & 6 & 4 & 7 \\
\hline Total & 99 & 32 & 38 & 24 & 36 \\
\hline
\end{tabular}

*Days.

Each streptococcal group comprised a reasonably constant proportion of the total numbers of bacteraemias during the observation period; there was a slight increase of GAS bacteraemias in the late 1980s. Of the GAS, 72 were T-typed: T- 1 accounted for $32 \%$, $\mathrm{T}-3,13, \mathrm{~B} 3264$ for $17 \%$, and T-12 and T-28 for $14 \%$ each. During 1988-1989 nearly half the isolates were T-1, compared with a mean figure of $23 \%$ for the $13-$ year survey period. During a 6-month period in the beginning of 1992 nearly all GAS bacteraemias were due to T-3,13,B3264. In GBS bacteraemias in adults, of the $39 \%$ of isolates that could be serotyped, types I, II and III were represented almost equally. GBS bacteraemia in neonates occurred with a mean annual number of 3 (range $0-8$ ); the majority $(87 \%$ ) were of the early onset type, i.e. occurring at the age of 5 days or less. Serotypes were identified in $87 \%$ of the isolates and serotypes I and III formed the majority of GBS bacteraemias in neonates.

The epidemiological data are shown in table II. Except for GBS bacteraemias in neonates there were no significant differences in patients with bacteraemia of the various streptococcal groups regarding sex, age, proportion of nosocomial cases, and mortality, although males predominated in GGS bacteraemias. According to our definition, $88 \%$ of GBS bacteraemias in neonates were nosocomial. The annual age-related incidences are listed in table III. The highest incidence of GBS bacteraemia $(8 \cdot 1 / 100000 /$ year) was in infants, and of GAS bacteraemia (4.1) in patients $>60$ years old; GCS and GGS bacteraemias were not recorded in patients under 18 years old.

Cardiopulmonary diseases were the most frequent predisposing factors in patients with GAS, GCS or GGS bacteraemia, but operative procedures were also prominent in GAS bacteraemias (table IV). Diabetes mellitus was the most frequent associated disease in adult patients with GBS bacteraemia.

The skin was the most frequent primary focus in GAS, GCS and GGS bacteraemia whereas urinary tract infection was most common in GBS bacteraemia 
Table III. Age-related incidences of $\beta$-haemolytic streptococcal bacteraemia in the County of Northern Jutland 1981-1993

\begin{tabular}{|c|c|c|c|c|c|c|c|c|c|}
\hline \multirow{2}{*}{$\begin{array}{l}\text { Age group } \\
\text { (years) }\end{array}$} & \multirow{2}{*}{$\begin{array}{l}\text { Mean population } \\
\quad(1981-1993)\end{array}$} & \multicolumn{2}{|c|}{ GAS } & \multicolumn{2}{|c|}{ GBC } & \multicolumn{2}{|c|}{ GCS } & \multicolumn{2}{|c|}{ GGS } \\
\hline & & Total & $\begin{array}{c}\text { Annual } \\
\text { incidence* }\end{array}$ & Total & $\begin{array}{c}\text { Annual } \\
\text { incidence* }\end{array}$ & Total & $\begin{array}{c}\text { Annual } \\
\text { incidence* }\end{array}$ & Total & $\begin{array}{c}\text { Annual } \\
\text { incidence* }\end{array}$ \\
\hline $0-4$ & 28275 & 7 & $1 \cdot 8$ & 32 & $8 \cdot 1$ & 0 & $0 \cdot 0$ & 0 & 0.0 \\
\hline $5-9$ & 30128 & 5 & $1 \cdot 2$ & 0 & $0 \cdot 0$ & 0 & 0.0 & 0 & 0.0 \\
\hline $10-19$ & 70330 & 1 & $0 \cdot 1$ & 1 & $0 \cdot 1$ & 1 & $0 \cdot 1$ & 0 & $0 \cdot 0$ \\
\hline $20-60$ & 260754 & 32 & 0.9 & 15 & 0.4 & 12 & $0 \cdot 3$ & 15 & 0.4 \\
\hline$\geqslant 61$ & 94423 & 54 & $4 \cdot 1$ & 22 & $1 \cdot 7$ & 11 & 0.8 & 21 & $1 \cdot 6$ \\
\hline
\end{tabular}

*Cases/100000 population/year.

Table IV. Predisposing factors in patients* with $\beta$-haemolytic streptococcal bacteraemia in the County of Northern Jutland 1981-1993

\begin{tabular}{lrrrrr}
\hline \multirow{2}{*}{$\begin{array}{l}\text { Predisposing } \\
\text { factor }\end{array}$} & \multicolumn{5}{c}{ Number of cases of bacteraemia with } \\
\cline { 2 - 6 } & GAS & GBS $\dagger$ & GCS & GGS & Total \\
\hline $\begin{array}{l}\text { Diabetes mellitus } \\
\text { Cardiovascular and }\end{array}$ & 6 & 11 & 2 & 3 & 22 \\
pulmonary diseases & 17 & 4 & 8 & 10 & 39 \\
Alcoholism & 6 & 1 & 1 & 3 & 11 \\
AlDS & 0 & 0 & 0 & 1 & 1 \\
Solid tumour & 6 & 5 & 1 & 3 & 15 \\
Haematological tumour & 1 & 2 & 4 & 5 & 12 \\
Steroid therapy & 11 & 1 & 3 & 5 & 20 \\
$\begin{array}{l}\text { Operative procedures } \\
\text { Miscellaneous }\end{array}$ & 16 & 5 & 4 & 2 & 27 \\
None & 21 & 5 & 3 & 5 & 34 \\
& 32 & 6 & 3 & 6 & 47 \\
\hline
\end{tabular}

*Some patients had more than one underlying condition.

†GBS bacteraemia in neonates not included.

Table V. Primary focus of infection in patients with $\beta$ haemolytic streptococcal bacteraemia in the County of Northern Jutland 1981-1993

\begin{tabular}{|c|c|c|c|c|}
\hline \multirow{2}{*}{$\begin{array}{l}\text { Primary } \\
\text { focus }\end{array}$} & \multicolumn{4}{|c|}{$\begin{array}{l}\text { Number of cases of } \\
\text { bacteraemia with }\end{array}$} \\
\hline & GAS & GBS* & GCS & GGS \\
\hline Wound/cellulitis & $30(12)$ & $5(2)$ & $5(3)$ & $17(4)$ \\
\hline $\begin{array}{l}\text { Osteomyelitis or } \\
\text { arthritis }\end{array}$ & $7(1)$ & $1(0)$ & $1(1)$ & $1(0)$ \\
\hline Pneumonia & $7(2)$ & $1(1)$ & $1(0)$ & $2(1)$ \\
\hline Pharyngitis & $4(4)$ & $0(0)$ & $1(1)$ & $3(3)$ \\
\hline Meningitis & $1(0)$ & $0(0)$ & $3(2)$ & $0(0)$ \\
\hline Endocarditis & $2(0)$ & $4(0)$ & $2(0)$ & $2(2)$ \\
\hline $\begin{array}{l}\text { Genital puerperal } \\
\text { infection }\end{array}$ & $9(9)$ & $3(2)$ & $1(0)$ & $0(0)$ \\
\hline $\begin{array}{l}\text { Urinary tract } \\
\text { infection }\end{array}$ & $0(0)$ & $6(5)$ & $2(2)$ & $0(0)$ \\
\hline Miscellaneous & $8(4) \dagger$ & $0(0)$ & $2(0) \ddagger$ & $0(0)$ \\
\hline No evident focus & 31 & 18 & 6 & 11 \\
\hline
\end{tabular}

Numbers in parentheses indicate cases verified bacteriologically. * GBS bacteraemia in neonates not included.

†One case each of sinusitis, abscess in gluteal muscles, necrotising fasciitis and infected haematoma; four cases of peritonitis. $\ddagger$ One case each of peritonitis and salpingitis.

in adults (table V). Puerperal infection was prominent in GAS bacteraemia. This did not appear in clusters. In children with GAS bacteraemia the most common foci were osteomyelitis or arthritis. The mortality rates of GAS, neonatal GBS, adult GBS, GCS and GGS bacteraemia were $23 \%, 28 \%, 16 \%, 17 \%$ and $19 \%$, respectively (table II). With the exception of GBS bacteraemias, the highest mortality rates were noted in patients $>60$ years old $(33 \%, 27 \%$ and $24 \%$ for GAC, GCC and GGS bacteraemia, respectively). Of the 23 cases of fatal GAS bacteraemia, 57\% died sooner than $24 \mathrm{~h}$ after blood culture. The corresponding figures for GGS bacteraemias and neonates with GBS bacteraemia were $43 \%$ and $78 \%$. However, deaths of patients with GCS bacteraemia or adults with GBS bacteraemia occurred only after $48 \mathrm{~h}$. In case of skin infection, $27 \%$ of patients with GAS bacteraemia died but none with GBS, GCS or GGS bacteraemia.

\section{Discussion}

An increase in GAS bacteraemia was reported in the late 1980s in Denmark, in line with other Scandinavian countries. ${ }^{1-3} \mathrm{~T}-1$ temporarily became the dominant $\mathrm{T}$ type in the County of Northern Jutland, conforming to the national trend. ${ }^{2}$ Continuous shifts in the dominant $\mathrm{M}$ - and T-types among GAS are considered to contribute to changes in morbidity, ${ }^{12.13}$ and in the survey area a marked but transient increase of $\mathrm{T}$ 3,13,B3264 strains was noticed during 1992. The survey confirms previous reports that GAS most frequently cause bacteraemia whereas GCS are the least frequent..$^{8,15-17}$ Contrary to other reports ${ }^{7.8}$ no increase in GBS bacteraemia in adults and GGS bacteraemia was found during 1981-1993. This may be related to differences in the study population regarding socio-economic status.

The finding that GCS and GGS bacteraemias were most frequent in elderly people, unlike GAS bacteraemia that occurred in all decades of life, agreed with previous studies. ${ }^{1,18-20}$ However, the incidence of $1 \cdot 8 / 100000 /$ year among children $<5$ years old was less than that reported elsewhere. ${ }^{1,16}$

With the exception of GBS bacteraemia in neonates, the rate of nosocomially acquired bacteraemias among the $\beta$-haemolytic streptococci was similar. The finding that $38 \%$ of GAS bacteraemias were nosocomial is in agreement with a nationwide Danish study ${ }^{21}$ but is higher than noted previously. ${ }^{8.16}$ One explanation may 
be that the definition of nosocomial infection applied ${ }^{11}$ is broader than in most of the above reports. Operative procedures were frequent in nosocomial GAS and GCS bacteraemia and GBS bacteraemia in adults; nearly half of the nosocomial GAS bacteraemias were related to orthopaedic, neurosurgical or gynaecological operations. The relevance of operative procedures in GAS bacteraemia has been reported previously ${ }^{8.21}$ and underlines the potential for GAS to act as nosocomial pathogens. Operative procedures related to nosocomial GGS bacteraemias were not common, as noted elsewhere. ${ }^{8}$

As in other reports, ${ }^{i}{ }^{18-19}$ GCS and GGS bacteraemia, and GBS bacteraemia in adults, occurred in patients with debilitating diseases such as diabetes mellitus and cancer, whereas GAS bacteraemias were not so frequently related to serious underlying diseases.

In agreement with other reports, the skin was the most common focus in GAS, GCS and GGS bacteraemia. ${ }^{8.15-17}$ An association has been reported between GGS bacteraemia and endocarditis. ${ }^{22}$ During the 13-year observation period, two cases of GGS endocarditis were recorded, one case was fatal within $72 \mathrm{~h}$. Wound infection and urinary tract infection have been listed as common foci of GBS bacteraemias in adults $^{7}$ but in this survey a primary focus could not be found in the majority of the cases, unlike other $\beta$ haemolytic streptococci.

Mortality rates of between $20 \%$ and $48 \%$ in GAS bacteraemia, that increased with age, have been reported. ${ }^{16.17 .20}$ This agrees with mortality rates of $8 \%$ in children $<10$ years old and $33 \%$ in patients $>60$

\section{References}

1. Martin PR. Høiby EA. Streptococcal serogroup A epidemic in Norway 1987-1988. Scand J Infect Dis 1990; 22; 421-429.

2. Henrichsen J. Rønne T. Reemergence of severe group A streptococcal infections in Denmark 1988-89. Zentralbl Bakteriol 1992: Suppl 22:8-10.

3. Strömberg A, Romanus V, Burman LG. Outbreak of group A streptococcal bacteremia in Sweden : an epidemiologic and clinical study. J Infect Dis 1991; 164: 595-598.

4. Centers for Disease Control. Group A beta-hemolytic streptococcal bacteremia - Colorado, 1989. MMWR 1990; 39: 36,11

5. PHLS Communicable Disease Surveillance Centre. Invasive group A streptococcal infections in Gloucestershire. Communicable Disease Report Weekly 1994; 4: 97.

6. Hasseltvedt V, Iversen B. Aavitsland P, Aasen S, Lystad A, Høiby EA. Alvorlige GAS-infeksjoner i 1993-94 opdatering (Serious GAS infections during 1993-94-an update). Oslo: National Institute of Public Health, Meldesystem for Infektsjonssykdommer 1994; 22 (21).

7. Farley MM. Harvey RC, Stull T et al. A population-based assessment of invasive disease due to group B streptococcus in nonpregnant adults. $N$ Engl J Med 1993; 328: 1807-1811.

8. Skogberg K. Simonen H, Renkonen O-V, Valtonen VV. Betahaemolytic Group A, B, C and G streptococcal septicaemia: a clinical study. Scand J Infect Dis 1988; 20: $119-125$.

9. Bruun B. Sloth K, Bentzon MW, Frederiksen W. A study of bacteremias in Denmark from 1977-1978. APMIS 1982; B 90: $309-317$. years old in this study. The mortality rate for the other groups of $\beta$-haemolytic streptococci was at least twice as high in patients $>60$ years of age than that in patients $20-60$ years old. The suggestion that GGS bacteraemia is a more benign disease than GAS bacteraemia ${ }^{8}$ was not confirmed in this study.

The incidence of GBS bacteraemia in neonates, $0 \cdot 4 / 1000$ live births, is lower than reported elsewhere. ${ }^{?}$ Nine neonates with GBS bacteraemia died, all of whom had early onset disease. In the County of Northern Jutland, only one of 15 neonates with GBS bacteraemia died in the period 1988-1993, in contrast with eight of 17 who died during 1981-1987. This may indicate a more favourable prognosis of neonatal GBS bacteraemia in recent years.

In conclusion, the proportion of GAS, GCS, GGS and GBS bacteraemias was fairly constant over a 13year period. GAS occurred in all decades of life, whereas GBS was found mainly in neonates and the elderly. GCS and GGS bacteraemias were mainly found in elderly patients. Except for GBS bacteraemia in neonates, there were no significant differences amongst the various $\beta$-haemolytic streptococci regarding age, sex, number of nosocomial isolates and mortality. Surgical intervention was an important predisposing factor in patients with GAS bacteraemia and this underlines the potential for GAS to act as nosocomial pathogens. Although there were no differences in mortality rates among the four bacterial groups, the majority of deaths of patients with GAS bacteraemia occurred within $24 \mathrm{~h}$ after blood cultures were obtained. This emphasises that GAS bacteraemia, although uncommon, is a serious event.

10. Prag J, Jensen J, Lebech $\mathbf{K}$. Darkening of haemoglobin in simulated, continuously agitated aerobic blood cultures: an early indicator of bacterial growth. APMIS 1991; 99: 1083-1088.

11. Garner JS, Jarvis WR, Emeri TG, Horan TC, Hughes JM. CDC definitions for nosocomial infections 1988. Am J Infect Control 1988; 16: 128-140.

12. Schwartz B. Facklam RR, Breiman RF. Changing epidemiology of group A streptococcal infection in the USA. Lancet $1990 ; 336$ : $1167-1171$.

13. Colman G, Tanna A, Efstratiou A, Gaworzewska ET. The serotypes of Streptococcus pyogenes present in Britain during 1980-1990 and their association with disease. $J \mathrm{Med}$ Microbiol 1993; 39: 165-178.

14. Schønheyder HC, Kristensen B, Højbjerg T, Hyttel L, Korsager B. T-types among group A beta-haemolytic streptococci in Denmark. J Hosp Infect 1993; 23 : 71-72.

15. Barnham M. Invasive streptococcal infections in the era before the acquired immune deficiency syndrome: a 10 years' compilation of patients with streptococcal bacteraemia in North Yorkshire. J Infect 1989; 18: 231-248.

16. Francis J, Warren RE. Streptococcus pyogenes bacteraemia in Cambridge-a review of 67 episodes. $Q J$ Med $1988 ; 68$ : 603-613.

17. Nielsen SV, Kolmos HJ. Bacteraemia due to different groups of beta-haemolytic streptococci: a two-year survey and presentation of a case of recurring infection due to Streptococcus "equisimilis". Infection 1993; 6: 358-361.

18. Auckenthaler R, Hermans PE, Washington JA. Group G streptococcal bacteremia: clinical study and review of the literature. Rev Infect Dis. 1983; 5: 196-204. 
19. Bradley SF, Gordon JJ, Baumgartner DD, Marasco WA, Kauffman CA. Group C streptococcal bacteremia : analysis of 88 cases. Rev Infect Dis 1991; 13: 270-280.

20. Bucher A, Martin PR, Høiby EA et al. Spectrum of disease in bacteraemic patients during a Streptococcus pyogenes serotype M-1 epidemic in Norway in 1988. Eur J Clin Microbiol Infect Dis 1992; 11: 416-426.
21. Strøbæk S, Jepsen OB, Zimakoff J, Rønne T. Increased number of sporadic nosocomial group A streptococcal bacteraemias during a community epidemic. $J$ Hosp Infect 1991; 19: 129-136.

22. Bucher A, Gaustad P. Septicemia and endocarditis caused by group G streptococci in a Norwegian hospital. Eur J Clin Microbiol Infect Dis 1990; 9: 251-256. 